\title{
RECENT ADVANCES IN FABRY-PEROT-BASED REFRACTOMETRY UTILIZING GAS MODULATION FOR ASSESSMENT OF PRESSURE
}

\author{
M. Zelan ${ }^{1}$, I. Silander ${ }^{2}$, C. Forssén ${ }^{1,2}$, J. Zakrisson ${ }^{2}$, O. Axner ${ }^{2}$ \\ ${ }^{1}$ Measurement Technology, RISE Research Institutes of Sweden, SE-501 15 Borås, Sweden, martin.zelan@rise.se \\ ${ }^{2}$ Department of Physics, Umeå University, SE-901 87 Umeå, Sweden, \\ isak.silaner@umu.se, clayton.forssen@umu.se, johan.zakrisson@umu.se, ove.axner@umu.se
}

\begin{abstract}
:
Gas modulation refractometry (GAMOR) is a methodology that can mitigate the influence of fluctuations and drifts in Fabry-Pérot cavity-based refractometry. This paper presents a thorough description of its principles, what it enables, and its applicability. An overview of the latest results is presented, including the realization of a system based upon a cavity spacer made of Invar that allows for detection of $\mathrm{N}_{2}$ with sub-ppm precision, and a characterization procedure that allows for assessment of $\mathrm{N}_{2}$ with an accuracy at low-ppm levels.
\end{abstract}

Keywords: Refractometry, GAMOR, Pressure.

\section{INTRODUCTION}

Fabry-Pérot cavity (FPC) based refractometry is a sensitive technique for assessment of gas refractivity, density, pressure, and flow using laser technology. By measuring the refractivity, $n-1$, where $n$ is the index of refraction, and the temperature of a gas, the pressure can be calculated by the use of the LorentzLorenz equation and an equation of state [1].

Recent works have indicated that the technique also has the potential to replace current pressure standards, in particular in the $1 \mathrm{~Pa}$ to $100 \mathrm{kPa}$ range [2]. Such a realization of the Pascal would not depend on any mechanical actuator but instead directly measure the gas density, potentially decreasing uncertainties and shortening calibration chains. With the revision of the SI-system in May 2019, in which the Boltzmann constant was given a fixed value [3], the performance would, in principle, be limited only by the accuracies of quantum calculations of gas parameters and assessments of the deformation of the cavity material and gas temperature.

The technique is built on the fact that refractive index constitutes the ratio of the optical length under two conditions; without and with gas in the measurement cavity, here referred to as $L_{0}$ and $L$ $\left(=n L_{0}\right)$, respectively. In practice, the refractive index is assessed by measuring the change in frequency of a laser, locked to a cavity mode, when gas with a known refractivity is let into the cavity.

Besides offering the possibility of outperforming conventional technologies, FPC-based refractometry has several other advantages. Due to the high accuracy of frequency measurements, and the fact that no moving parts are needed, the assessments can be done swiftly without any manual oversight.

Moreover, since the measurement principle is based on assessing a change in frequency, there is no fundamental limit of the dynamical working range of the technique. In practice, its lower limit is given by the resolution of the frequency measurement, while the upper limit is set by the mechanical properties of the cavity and the vacuum system. The fact that FPCbased refractometry can work with high precision over such a large pressure range, which currently requires several different techniques, makes it very versatile and unique.

The conventional means of assessing refractivity by the use of FPC-based refractometry is to assess $L_{0}$ and $L$ in two separate assessments. Under ideal conditions, under which no physical parameter of the system changes with time, it is possible to envision a procedure in which first, once and for all, $L_{0}$ is assessed with no gas in the cavity, denoted a reference measurement. Based on this, the refractivity (and thereby the pressure) can then be assessed by measurements of the optical length of the cavity under other conditions.

However, in practice, this is not trivial since the cavity spacer material is subjected to deformation due to gas pressure, thermal expansion, aging, relaxations, and diffusion of gas into the material that changes its length in unpredicted ways. For example, a change in length of a $30 \mathrm{~cm}$ long cavity of solely 1 pm (a fraction of the "size" of an atom) corresponds to an error in the assessment of pressure (for $\mathrm{N}_{2}$ ) of 1 $\mathrm{mPa}$. This severely restricts the performance and applicability of FPC-based refractometry to such an extent that its performance is in practice limited by the stability of the cavity length. 
The conventional way of minimizing these limitations is to construct the FPC of low thermal expansion glass, e.g. ultra-low expansion glass (ULE®) or Zerodur ${ }^{\circledR}$, place the refractometer in a highly temperature stabilized environment (combined gas and vacuum chamber), and let the system relax and equilibrate for long time periods after each gas filling/emptying. However, this is a cumbersome effort that increases the complexity of the systems as well as limits the use of the technology outside well-controlled laboratories.

To reduce the constraints of such requirements, we decided to develop a methodology that could reduce the effect of fluctuations and drifts of the length of the cavity on the assessments. The methodology, which is built on a regularly alteration of the amount of gas in an FPC, was originally referred to as drift free (DF) or fast switching (FS) dual FPC (DFPC) based refractometry, but is nowadays referred to as Gas Modulation Refractometry (GAMOR) [4-9].

This paper provides a simple and comprehensible description of this methodology. It also gives an overview of the recent technological achievements and presents results that have followed from the latest development of the methodology.

\section{THE PRINCIPLES OF GAS MODULATION REFRACTOMETRY}

GAMOR is based on the same fundamental principle as ordinary (unmodulated) refractometry; it measures the change in refractive index between two situations; a reference measurement without gas, and a measurement with gas, in the measurement cavity.
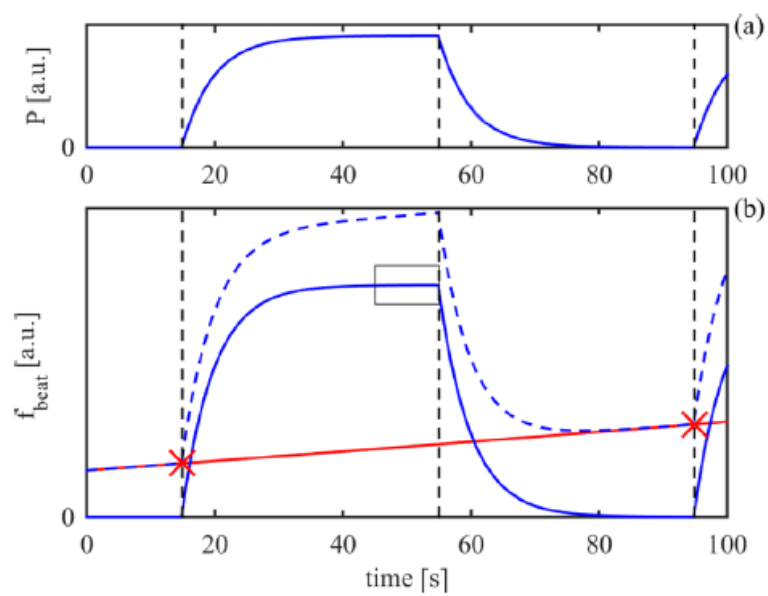

Figure 1: Illustration of the GAMOR-principle. Upper panel: the pressure in the measurement cavity during a cycle. Lower panel: illustration of the measurement of the corresponding shift in frequency (dashed-blue curve). By taking two reference values in rapid succession (red x) and making a linear interpolation between them (red line), linear drifts in the system can be eliminated (providing the solid curve). Reproduced with permission from Ref. [4].

However, in GAMOR, the amount of gas in the measurement cavity is modulated to allow for a large number of repeated measurements. This creates two cornerstones that GAMOR is built upon:

(i) The first one is based on the principle that the influence of drifts and fluctuations ${ }^{1}[9]$, in particular those thermally induced and those from aging, can be significantly (orders of magnitude) reduced by the performance of the frequent reference assessments in an empty cavity (marked with red crosses in Fig. 1);

(ii) The second one encompasses an interpolation procedure in which the response of the empty cavity system can be estimated at all times of the filled cavity assessment (marked with the red line in Fig. 1). By this, the methodology can eliminate additional fluctuations as well as all remaining influences of the linear parts of the drifts.

This allows the technique to benefit, also at long time scales, from the high precision FPC-based refractometry has at short time scales.

\section{MEANS OF EVALUATION OF REFRACTIVITY, MOLAR DENSITY, AND PRESSURE}

GAMOR has so far been based on DFPC systems and is hence governed by the same basic equations as unmodulated DFPC-refractometry.

It has been found convenient to express the refractivity of the gas under scrutiny as a function of the shifts of the beat frequency between the two cavities, $\Delta f$, and the mode numbers of the modes addressed in the measurement cavity, $\Delta q_{1}$, when the latter is filled with (or emptied of) gas, as

$$
n-1=\frac{\overline{\Delta f}+\overline{\Delta q_{1}}}{1-\overline{\Delta f}+\varepsilon},
$$

where $\overline{\Delta f}$ is the shift of the relative beat frequency given by $\Delta f / v_{01}$, where $v_{01}$ is the empty cavity frequency of the measurement laser, and $\overline{\Delta q_{1}}$ is a shorthand notation for $\Delta q_{1} / q_{01}$, where $q_{01}$ is the number of the mode addressed in the empty measurement cavity. $\varepsilon$ is a normalized relative cavity deformation, defined as $\left(\delta L / L_{0}\right) /(n-1)$, where $\delta L$ and $L_{0}$ are the pressure induced length deformation and the empty cavity length, respectively $[4,5]$.

For pressure up to atmospheric pressure, the gas density $\rho$ can be calculated from the refractivity by use of the extended Lorentz-Lorenz equation,

$\rho=\frac{2}{2 A_{R}}(n-1)\left[1+b_{n-1}(n-1)\right]$,

\footnotetext{
${ }^{1}$ In particular fluctuations that appear on time scales of the measurement cycle or longer.
} 
where $A_{R}$ and $b_{n-1}$ are the molar dynamic polarizability and a series expansion coefficient, respectively. The latter is given by $-\left(1+4 B_{R} / A_{R}^{2}\right) / 6$, where, in turn, $B_{R}$ is the second refractivity virial coefficient in the Lorentz-Lorenz equation $[5,10]$.

Up to atmospheric pressure, the pressure can be obtained from the density and temperature as

$$
P=R T \rho\left[1+B_{\rho}(T) \rho\right],
$$

where $R$ is the ideal gas constant, $T$ is the temperature of the gas, and $B_{\rho}(T)$ is the second density virial coefficient $[5,10]$.

If higher pressures are to be assessed, additional higher order terms need to be added in the Eqs. (2) and (3).

It is of importance to note that Eq. (1) shows that the refractivity (and thereby the molar density and pressure) can be assessed without any monitoring or assessment of $L_{0}$. There is neither any need to explicitly assess the free-spectral range (FSR). The reason no knowledge of $L_{0}$ is needed is due to the use of frequent reference assessments, which repeatedly monitor the combined status of the two empty cavities, as well as the fact that $\varepsilon$ can be assessed experimentally [11]. The reason no assessment of the FSR is needed is that $\overline{\Delta q_{1}}$ is not expressed in terms of the FSR, but instead in the mode number of the cavity mode addressed, $q_{01}$. This is an integer that can be assessed uniquely (i.e. with no uncertainty) by ensuring that the assessed refractivity is a continuous function when the measurement laser is making a (controlled) mode hop [11].

\section{TYPICAL SET-UPS}

Over the years, a variety of GAMOR instrumentation and procedures have been developed [4-7, 11]. While the first versions utilized a simplistic design and were constructed to carry out proof-of-principle demonstrations [4], the later realizations have been gradually upgraded to improve on their performance, firstly precision $[5,6]$ and lately accuracy $[7,11]$. To visualize the developments during the last few years, Fig. 2 displays a collection of pictures of some GAMOR-based refractometers.

Common for all systems is that they have been based on DFPC systems with FSRs of around $1 \mathrm{GHz}$ and finesse values of $10^{4}$. Each cavity is probed by an Erdoped fiber laser emitting light within the $\mathrm{C} 34$ communication channel, i.e., around $1.55 \mu \mathrm{m}$, that is coupled into an acousto-optic modulator (AOM) for fast locking feedback and an electro-optic modulator (EOM) modulated at $12.5 \mathrm{MHz}$ for Pound-Drever-Hall locking.

For the assessment of pressure, the measurement of the gas temperature is crucial. In most GAMOR instrumentation, the gas has been monitored by Pt-100 sensors placed in holes drilled into the cavity spacer. However, from our experience, even when the sensors have been properly calibrated, they constitute a limiting
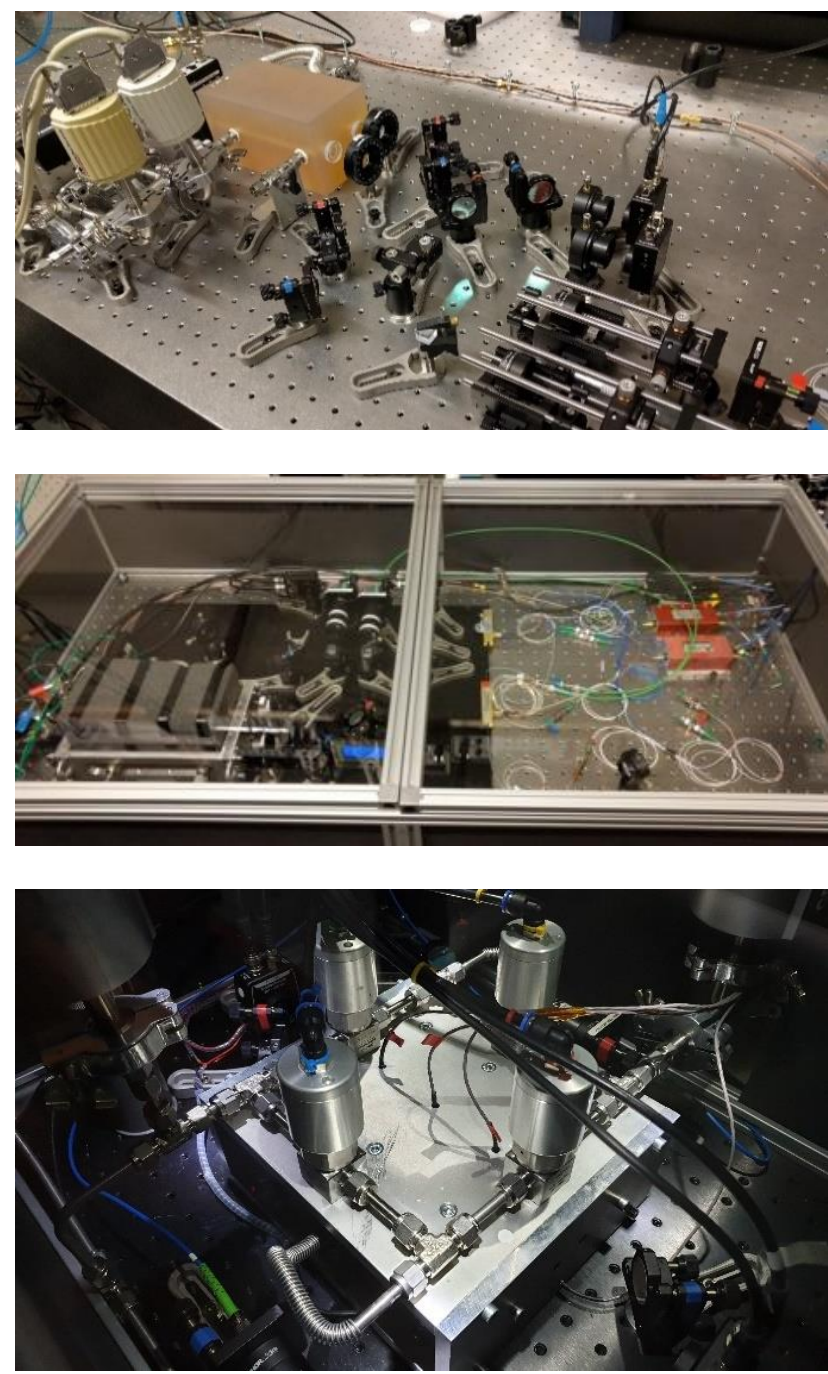

Figure 2: Pictures of the GAMOR refractometer-system during various stages of the development. The top pictures show one of the earliest versions using a non-temperature stabilizes Zerodur-cavity and plenty of free-space optics. The middle images show a later version of the same system, now placed in a box for temperature stabilization and using less free-space optics and more fiber-optics. The bottom pictures show the most recent version of the GAMOR-system consisting of an Invar-cavity encapsulated in an aluminium oven.

factor in terms of accuracy. Therefore, as is described in some detail in an accompanying presentation [7], we have recently constructed a Ga fixed-point cell that, by the use of a thermocouple working close to zero temperature difference, allows us to assess the temperature of the FPC, and hence the gas inside it, at low ppm-levels.

\section{RESULTS AND PROGRESS}

\subsection{Experimental demonstration of the ability of GAMOR to reduce drifts}

An example of the powerfulness of the GAMOR methodology in reducing the influence of drifts is shown in Fig. 3 [4]. The upper blue curve in panel (a) 

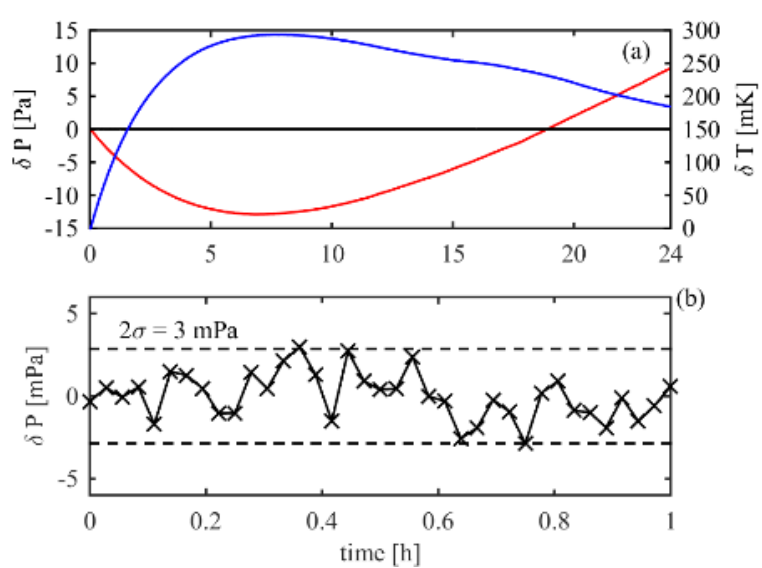

Figure 3: Illustration of the GAMOR-principle. Upper panel: a $24 \mathrm{~h}$ long series of measurement of an empty measurement cavity evaluated by two different means: the lowermost (red) curve - without gas modulation, and the almost fully horizontal (black) curve - by use of the GAMOR methodology (both left axis). The uppermost curve (blue, right axis) represents the temperature. Lower panel: a zoom in of the first hour section of the data taken with the GAMOR methodology. Note the differences in scales. Reproduced with permission from [4].

(right axis) shows the temperature drift over 24 hours while the lower red curve (left axis) shows the corresponding drift of a conventional non-temperaturestabilized DFPC refractometry signal (expressed in units of pressure) from an empty cavity.

The horizontal black line is not, as it might appear, the time axis, but is, in fact, the actual corresponding GAMOR signal. Panel (b) shows an enlargement of a part of this data. Note the more than three orders of magnitude differences in scales. This illustrates the ability of the GAMOR methodology to significantly reduce (in practice eliminate) the drifts that limit conventional DFPC-based refractometry [4].

\subsection{Investigation of the capability of GAMOR to reduce fluctuations}

In a recent work [9], we have provided a thorough explication of the capability of GAMOR to significantly reduce the effect of fluctuations. It explicates, in terms of the length of the modulation cycle, to which extent the modulation procedure can reduce the influence of fluctuations.

The upper panel in Fig. 4 illustrates the maximum fraction of specific Fourier components of fluctuations an unmodulated refractometry system (in which the time between the filled- and emptycavity measurements, $t_{\text {um }}$, is $10^{5} \mathrm{~s}$ ) picks-up as a function of its Fourier frequency, $f_{F}$. The lower panel displays the same entity when the detection system utilizes the first cornerstone of the GAMOR methodology, $(i)$, i.e. a frequent reference assessment (using a modulation cycle of $100 \mathrm{~s}$ ). A comparison of the two shows that the repeated reference assessment is capable of reducing the influence of fluctuations

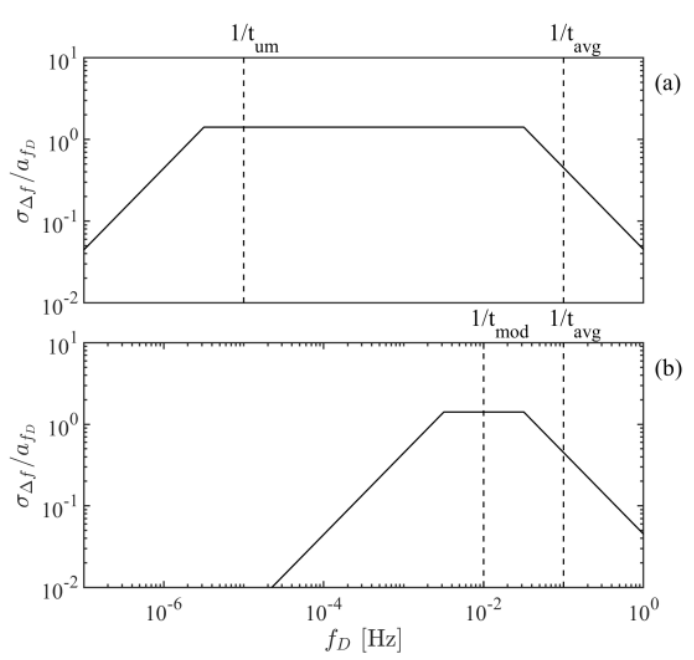

Figure 4: Illustration of the ability of the GAMORprinciple to reduce fluctuations. The maximum fraction of specific components of fluctuations the system picks-up as a function of its Fourier frequency in terms of their normalized rms values for two different lengths of the gas filling-and-emptying cycle. Panel (a) represents the case with unmodulated detection with a gas filling-andemptying time, $t_{u m}$, of $10^{5} \mathrm{~s}$ while panel (b) illustrates the situation with gas modulated detection, utilizing a gas modulation period, $t_{\text {mod }}$, of $10^{2} \mathrm{~s}$. In both cases, an averaging time, $t_{\text {avg }}$, of $10 \mathrm{~s}$ has been assumed.

with Fourier frequencies below $1 /\left(\pi t_{\text {mod }}\right)$ significantly [those below $1 /\left(\pi t_{u m}\right)$ three orders of magnitude].

As is shown in an upcoming work, the GAMOR methodology will also significantly reduce the influence of drifts. The first cornerstone of the methodology $(i)$ will convert linear drift to small amounts of inaccuracy. The interpolation part of the methodology, cornerstone (ii), will then eliminate this methodology-induced inaccuracy. It will additionally reduce the low frequency components; in the case considered, the influence of fluctuations with low Fourier frequencies [below $1 /\left(\pi t_{u m}\right)$ ] will be reduced up to six orders of magnitude. This makes GAMOR virtually insensitive to both slow fluctuations and drifts of the lengths of the cavities.

\subsection{Use of alternative cavity spacer materials}

Since GAMOR has the ability to reduce the influence of fluctuations and drift, it allows for the use of non-conventional cavity spacer material. As is described in $[6,7]$, we have recently developed a refractometer based on a cavity spacer made of Invar, which is a material that has a number of advantages over ULE® and Zerodur $\AA$, mainly: (1) a higher ratio of thermal conductivity to volumetric heat capacity; (2) a higher volumetric heat capacity; (3) a higher Young's modulus; (4) presumably a lower degree of He diffusivity; and (5) it can be machined in a metal workshop, whereby more complicated geometries can be created more swiftly at a lower cost.

Using a dead weight piston balance to provide a reference pressure, we have recently demonstrated 
that such a system can outperform a similar one made of Zerodur®. As is shown in Fig. 5, the system could, for an empty cavity, demonstrate a white noise response up to $10^{4} \mathrm{~s}$ with a minimum deviation (defined as the Allan deviation) of $0.03 \mathrm{mPa}$. When set to assess pressure at $4303 \mathrm{~Pa}$, the system could provide (for measurement times of $10^{3} \mathrm{~s}$, representing 10 measurement cycles) a minimum Allan deviation of $0.34 \mathrm{mPa}$, which corresponds to a relative deviation of $0.08 \mathrm{ppm}$. These results, which are a few times better than those obtained using a Zerodurbased cavity, can, to a large extent, be attributed to the advantageous thermal properties of Invar.

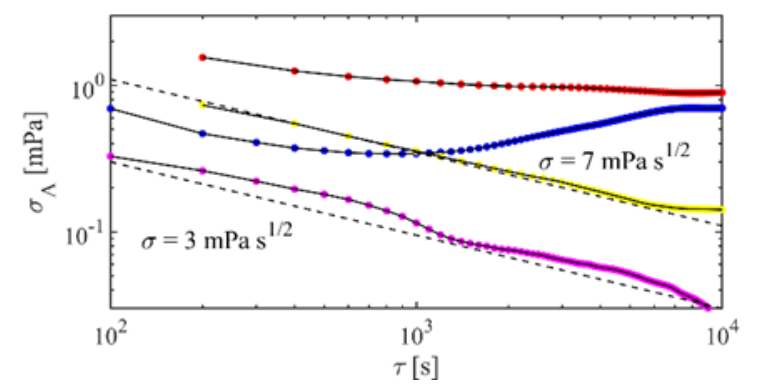

Figure 5: Allan deviations of pressure assessments made by the previous Zerodur-based system (red upper markers) and the novel Invar-based system (blue), both at $4303 \mathrm{~Pa}$. The yellow and purple (lower) markers illustrate empty cavity measurements using the same two systems, respectively. The figure is reproduced and modified with permission from [6].

\subsection{Procedure for assessing the pressure induced cavity deformation}

To assess pressure with adequate accuracy, a FPC-based refractometer system has to be characterized with respect to its pressure induced cavity deformation. To address this, we have, inspired by the work of Egan et al [12], recently developed a procedure for robust assessment of cavity deformation in FP-based refractometers that provides extraordinarily small amounts of uncertainty [11]. The procedure is not only insensitive to any systematic error in the set pressure, but also not influenced by a systematically incorrect assessment of the gas temperature. Furthermore, it is also insensitive to leakages of gas with the same refractivity as that addressed and outgassing processes.

The procedure is based on assessing the difference between the pressure assessed by the uncharacterized refractometer and a supplied reference pressure at a series of pressures for two gases with dissimilar refractivity (He and $\mathrm{N}_{2}$ ). By fitting linear curves to these responses, their slopes are used as a basis for the assessment of the pressure-induced deformation of the cavity. The procedure is also capable of providing an accurate assessment of the sum of systematic errors of the temperature assessment, the molar polarizabilities used, and the set pressure.
It is predicted that when a high-precision (subppm) refractometer is characterized, the uncertainty in the assessed deformation will contribute to the uncertainty in the assessment of pressure of $\mathrm{N}_{2}$ with solely a fraction of its uncertainty of the molar polarizability, presently $c a$. $1 \mathrm{ppm}$. This is a keyfinding for the further development of refractometry in general and GAMOR in particular, since it implies that cavity deformation no longer will be a limiting factor in FP based assessments of pressure of $\mathrm{N}_{2}$.

\subsection{Assessment of gas temperature}

As was alluded to above, to assess the gas temperature accurately, we have recently designed, constructed, and installed a gallium fixed-point cell based temperature reference system. The system can provide a stable temperature up to 60 hours. By operating the DFPC-system as close as possible to the temperature of the melting-point of $\mathrm{Ga}$, and measure the remaining temperature difference between the DFPC-cavity and the $\mathrm{Ga}$ system with a type-T thermocouple, we can assess the temperature of the cavity with an accuracy below $5 \mathrm{ppm}$. The design and preliminary results are presented in detail in an accompanying work [7].

\subsection{Dynamic range}

Since narrow linewidth lasers have a restricted tuning range, we have, in the realization of our GAMOR systems, implemented a mode jumping procedure by which the laser automatically jumps to a neighbouring mode if the shift of the mode addressed is larger than the tunability of the laser (which typically is a few FSRs of the cavity). This implies that there is, in practice, no upper limit for its dynamic range! Since the resolution is in the tens of $\mu \mathrm{Pa}$ range [6,7], given by stochastic noise in the system, and assessments have so far been demonstrated up to the tens of kPa range [5], a GAMOR based refractometry system is expected to have an extraordinary dynamic range (possibly up to 9 orders of magnitude).

\subsection{A transportable refractometer}

GAMOR has also opened up for a simplified realization of the experimental set-up and measurement environment. As is reported in an accompanying work [8], the reduced constrains have allowed for the construction of a transportable refractometer that is to be used for various future scientific endeavours, including a European circlecomparison.

\section{SUMMARY AND CONCLUSION}

This paper presents an overview of the current status of Fabry-Perot-cavity (FPC) based gas modulation refractometry (GAMOR) for pressure assessment. The basis of the GAMOR technique, including its most prominent advantages of reducing 
the influence of fluctuations and drifts of the length of the cavity spacer, have been explicitly explained.

The paper summarizes our most recent advances. One such is the use of cavity spacer material with advantageous properties compare to conventional spacer materials. In particular, we present results from a system with a FPC made of Invar. When assessing pressure at $4303 \mathrm{~Pa}$, the system could provide a minimum Allan deviation of $0.34 \mathrm{mPa}$, which corresponds to a relative deviation of $0.08 \mathrm{ppm}$. These results, which are a few times better than those previously obtained using a Zerodur-based cavity, can, to a large extent, be attributed to the advantageous thermal properties of Invar.

Furthermore, a procedure for assessing the pressure induced cavity deformation with such an accuracy that it does not contribute to the accuracy of the assessment of pressure with more than $1 \mathrm{ppm}$ [11], as well as a temperature measurement system based on the melting point of Ga [7], providing accuracy of below $5 \mathrm{ppm}$, are shortly summarized. This implies that neither cavity deformation, nor gas temperature, are the main limiting factors in FP based assessments of pressure of $\mathrm{N}_{2}$. Instead, the limiting factor is the knowledge of its molar refractivity, which presently is $7 \mathrm{ppm}$ [13].

The paper also explicitly emphasizes the potentially extraordinary large dynamical range of the technology that is made possible with automatic mode-jumping.

The paper finally notes that the GAMOR methodology allows for the realization of transportable instrumentation. A first such instrument has been constructed [8] and an improved version is under construction.

This project (QuantumPascal, 18SIB04) has received funding from the EMPIR programme cofinanced by the Participating States and from the European Union's Horizon 2020 research and innovation programme. The authors acknowledge support from the Swedish Research Council (VR) (Project No. 621-2015-04374); the Umeå University Industrial Doctoral School (IDS); the Vinnova Metrology Programme (Project Nos. 2018-04570 and 2019-05029); and the Kempe Foundations (Project No. 1823, U12).

\section{REFERENCES}

[1] P. F. Egan, J. A. Stone, J. H. Hendricks, J. E. Ricker, G. E. Scace, G. F. Strouse, Performance of a dual Fabry-Perot cavity refractometer, Opt. Lett., vol. 40, pp. 3945-3948, 2015.

[2] K. Jousten, J. Hendricks, D. Barker, K. Douglas, S. Eckel, P. Egan, J. Fedchak, J. Flugge, C. Gaiser, D. Olson, J. Ricker, T. Rubin, W. Sabuga, J. Scherschligt, R. Schodel, U. Sterr, J. Stone, G. Strouse, Perspectives for a new realization of the pascal by optical methods, Metrologia, vol. 54, pp. S146-S161, 2017.

[3] M. Stock, R. Davis, E. de Mirandés, M. J. T. Milton, The revision of the SI-the result of three decades of progress in metrology, Metrologia, vol. 56, p. 049502, 2019.

[4] I. Silander, T. Hausmaninger, M. Zelan, O. Axner, Gas modulation refractometry for high-precision assessment of pressure under non-temperaturestabilized conditions, J. Vac. Sci. Technol. A, vol. 36, p. 03E105, 2018.

[5] I. Silander, T. Hausmaninger, C. Forssén, M. Zelan, O. Axner, Gas equilibration gas modulation refractometry for assessment of pressure with subppm precision, J. Vac. Sci. Technol. B, vol. 37, p. 042901, 2019.

[6] I. Silander, C. Forssén. J. Zakrisson, M. Zelan, O. Axner, Invar-based refractometer for pressure assessments, Opt. Lett., vol. 45, pp. 2652-2656, 2020.

[7] I. Silander, C. Forssén, J. Zakrisson, M. Zelan, O. Axner, An Invar-based Fabry-Perot cavity refractometer with a gallium fixed-point cell for assessment of pressure, Acta IMEKO 9 (2020) 5.

[8] C. Forssén, I. Silander, D. Szabo, G. Jönsson, M. Bjerling, T. Hausmaninger, O. Axner, M. Zelan Transportable refractometer for assessment of pressure in the $\mathrm{kPa}$ range with ppm level precision, Acta IMEKO, 2020.

[9] O. Axner, I. Silander, C. Forssén, J. Zakrisson, M. Zelan, The ability of gas modulation to reduce the pick-up of fluctuations in refractometry, JOSA B, vol. 37, pp. 1956-1965, 2020.

[10] A. D. Buckingham, C. Graham, Density dependence of refractivity of gases, P. Roy. Soc. Lond. A Mat., vol. 337, pp. 275-291, 1974.

[11] J. Zakrisson, I. Silander, C. Forssén, M. Zelan, O. Axner, Procedure for robust characterization of Fabry Perot based refractometer, J. Vac. Sci. Technol. B, vol 38, p. 054202, 2020

[12] P. F. Egan, J. A. Stone, J. K. Scherschligt, and A. H. Harvey, Measured relationship between thermodynamic pressure and refractivity for six candidate gases in laser barometry, J. Vac. Sci. Technol. A37, 031603 (2019)

[13] P. F. Egan, J. A. Stone, J. E. Ricker, J. H. Hendricks, Comparison measurements of low-pressure between a laser refractometer and ultrasonic manometer, Rev. Sci. Instrum. vol. 87, p. 053113, 2016. 